Facial Redness Increases Men's Perceived Healthiness and Attractiveness

Christopher A. Thorstenson*1,2

Adam D. Pazda ${ }^{3}$

Andrew J. Elliot ${ }^{1}$

David I. Perrett ${ }^{4}$

Word Count: 5241

* Corresponding author, c.thorstenson@rochester.edu

${ }^{1}$ Department of Clinical and Social Sciences in Psychology, University of Rochester, 500 Wilson Blvd, Rochester, NY 14627, USA

${ }^{2}$ Munsell Color Science Laboratory, Rochester Institute of Technology, 1 Lomb Memorial Drive, Rochester, NY 14623, USA

${ }^{3}$ Department of Psychology, University of South Carolina - Aiken, 471 University Parkway, Aiken, SC 29801, USA

${ }^{4}$ School of Psychology \& Neuroscience, University of St. Andrews, Westburn Lane, St Andrews, Fife, KY16 9JP, United Kingdom 


\begin{abstract}
Past research has shown that peripheral and facial redness influences perceptions of attractiveness for men viewing women. The current research investigated whether a parallel effect is present when women rate men with varying facial redness. In four experiments, women judged the attractiveness of men's faces, which were presented with varying degrees of redness. We also examined perceived healthiness and other candidate variables as mediators of the redattractiveness effect. The results show that facial redness positively influences ratings of men's attractiveness. Additionally, perceived healthiness was documented as a mediator of this effect, independent of other potential mediator variables. The current research emphasizes facial coloration as an important feature of social judgments.
\end{abstract}

Keywords: facial coloration, red, attractiveness, healthiness, social perception 
Facial Redness Increases Men's Perceived Healthiness and Attractiveness

Recent research has shown that heterosexual men are more likely to rate a woman as more attractive when she is displaying red (e.g., when surrounded by red, holding something red, or wearing red clothing or lipstick; Elliot \& Niesta, 2008; Gilston \& Privitera, 2016; Guéguen, 2012a, 2012b; Guéguen \& Jacob, 2012a, 2012b; Lin, 2014; Pazda, Elliot, \& Greitemeyer, 2014; Robert, Havilcek, \& Owen, 2010; Stephen \& McKeegan, 2010; Young, 2015). These findings are presumably due, in part, to a pervasive link between red and romance across time and cultures. For instance, the color red is associated with love, sex, passion, and fertility across diverse cultures, dating back at least 10,000 years. (Hutchings, 2004; Knight, Power, \& Watts, 1995; Ragas \& Kozlowski, 1998). However, these red-romance links are primarily femalespecific.

Some work exists that examines the effect of red on women's perceptions of men's attractiveness (Elliot et al., 2010; Roberts et al., 2010), although research in this direction is less common, and a parallel effect is not always observed (Elliot \& Maier, 2013; Hesslinger, Goldbach, \& Carbon, 2015) ${ }^{1}$. The effect of red on ratings of women's attractiveness may be more robust because ratings of men's attractiveness are more likely to be moderated by the targets' emotion expression, women's conception risk, and perceptions of aggression or anger (Buechner, Maier, Lichtenfeld, \& Elliot, 2015; Prokop, Pazda, \& Elliot, 2015; Stephen, Oldham, Perrett, \& Barton, 2012; Young, Elliot, Feltman, \& Ambady, 2013). Facial redness is a sexually dimorphic characteristic; male faces are typically redder than female faces (Frost, 2005; Edwards \& Duntley, 1939), and people use this information when categorizing the sex of faces (Tarr,

\footnotetext{
${ }^{1}$ A study by Lynn, Giebelhausen, Garcia, Li, and Pauumanon (2013) did not show a positive red effect, but this study established an achievement context (not a romantic context) and even provided mixed achievement feedback (describing the performance of the target as "good but not exceptional", p. 3). Thus, based on theory (color-in-context theory, Elliot \& Maier, 2012) and prior research (Maier et al., 2013), a positive effect of red would not be expected (this may particularly be the case for women responding to men, given that women value high status, including competence, in those of the opposite sex; Buss, 2015).
} 
Kersten, Cheng, \& Rossion, 2010; Nestor \& Tarr, 2008). Since sexually dimorphic cues contribute to attractiveness in general (Rhodes, 2006), it follows that male facial redness should lead to perceptions of attractiveness. Taken together, it seems that red influences women's evaluations of men under some conditions but not others. The current research further investigates whether red affects men's attractiveness, and if so, the mechanism behind such evaluations. Instead of focusing on peripheral red cues (e.g., background or clothing), we focus on facial redness.

Faces convey a range of important social information, partly through color. Faces naturally change color as a consequence of physiological and psychological processes linked to persistent (e.g., cardiovascular health) and transient (e.g., emotion expression) conditions (see Changizi \& Shimojo, 2011). While facial coloration changes are very subtle, recent research suggests that human vision is particularly effective at detecting them (Changizi, Zhang, \& Shimojo, 2006; Tan \& Stephen, 2013; although see Burriss et al., 2015, who found that changes in female facial redness across the ovulatory cycle may not be detectable). Surprisingly, there is very little existing research examining the influence of men's facial redness on their perceived attractiveness when viewed by women. Recent studies revealed that both male and female facial color influenced their attractiveness (Re, Whitehead, Xiao, \& Perrett, 2011; Carrito et al., 2016, Stephen et al., 2012, Said \& Todorov, 2011), but the raters in these samples consisted of both men and women, and investigating the mechanisms behind such effects was not the central focus of this work.

Facial redness is indicative of healthiness for several reasons. Physical fitness is positively related to vascularization and blood oxygenation of the skin, making it appear redder (Armstrong \& Welsman, 2001; Johnson, 1998; Stephen, Coetzee, Law Smith, \& Perrett, 2009). 
Conversely, diabetes, hypertension, and cardiac illness impair the skin's vascularization and blood oxygenation, making it appear less red (Changizi \& Shimojo, 2011; Charkoudian, 2003; Panza, Quyyumi, Brush, \& Epstein, 1990; Ponsonby, Dwyer, \& Couper, 1997). Further, there is some empirical evidence that people associate increased facial redness with increased apparent health (Pazda, Thorstenson, Elliot, \& Perrett, 2016; Re et al., 2011; Stephen, Law Smith, Stirrat, \& Perrett, 2009; Stephen, Coetzee, Law Smith, \& Perrett, 2009; Jones, Porcheron, Sweda, Morizot, \& Russell, 2016). Therefore, in the current research we expected that facial redness would positively influence women's perceptions of men's healthiness.

Perceived healthiness likely contributes to perceptions of attractiveness because healthiness is a predictor of mate quality and reproductive potential (Perrett, 2010; Rhodes, 2006). Accordingly, we expected that women's perceptions of men's healthiness would positively predict their perceptions of men's attractiveness, and that the effect of facial redness on men's perceived attractiveness would be mediated by perceived healthiness.

\section{Overview of the present research}

We conducted four experiments that directly tested whether the red-attractiveness effect that has been documented for men viewing women is present in the opposite direction (i.e., for women viewing men) when facial redness is manipulated. Specifically, we manipulated face color by either increasing or decreasing the baseline facial redness on pictures of men, and then asked women to judge the men's attractiveness. We expected that facial redness would positively influence women's perceptions of the men's attractiveness. Additionally, we investigated whether healthiness perceptions mediated this relation.

In Experiments 1 and 2, female participants were asked to choose which of two simultaneously-presented male faces with varying amounts of redness was more attractive and 
sexually attractive. In Experiment 3, female participants rated the healthiness and attractiveness of male faces with varying amounts of redness, which allowed us to test perceived healthiness as a variable mediating the effect of facial redness on attractiveness. In Experiment 4, female participants again rated the healthiness and attractiveness of male faces with varying amounts of redness, as well as other candidate mediator variables (i.e., honesty, intelligence, and political conservatism) in order to determine whether perceived healthiness was a unique mediator of the red-attraction relation.

\section{Experiment 1}

In Experiment 1, we focused on whether women would find men with increased facial redness (relative to decreased facial redness) more attractive. We utilized a forced choice design, whereby women were instructed to choose which of two simultaneously presented faces (with varying degrees of redness) was more attractive and sexually attractive.

\section{Method}

Participants. One hundred and eight women (mean age $=31.4$, range $=19-64)$ were recruited from Amazon's Mechanical Turk and paid \$.10 for participating. Our sample was not restricted by geographic area, though most participants $(80 \%)$ were from the United States. Our sample size in this and all subsequent experiments was determined by assuming a small-tomoderate effect size (Cohen's $d=.35$ ) and obtaining enough participants to achieve a minimum of $80 \%$ power $(n=67$, which we were able to exceed by a large margin). Participation in this and all subsequent experiments was restricted a priori to heterosexual and bisexual individuals without a color-vision deficiency.

Procedure. In each experiment, all data were collected before any analyses were conducted, and all data exclusions, manipulations (i.e., experimental conditions), and variables 
analyzed are reported. Participants began the online study, which was described as a task in which they would rate human faces on various characteristics, by clicking on a web link. They completed six trials whereby they were instructed to select which of two simultaneously presented faces was more attractive. In each trial, the faces were of the same target, but one version had increased facial redness, and the other had decreased facial redness. The faces were displayed side-by-side, and each version was randomly assigned to be displayed on the left or right side of the screen. Each trial consisted of a different pair of target faces (yielding six male targets in total). The order of presentation for the six face identities was randomized. After completing trials for all six faces, the procedure was repeated, but participants were instructed to select the face that they found more sexually attractive.

Stimuli. The stimuli consisted of six male target faces. Each face was a composite of three moderately attractive, young Caucasian men. Color masks were applied to the skin areas of the faces (i.e., excluding the eyes and hair) using Matlab. We then manipulated the redness of each face $\left(a^{*}\right)$ in CIELAB color space by +5 or -5 units, using Psychomorph (see Stephen, Law Smith, Stirrat, \& Perrett, 2009, for a detailed example of how facial color information can be manipulated in this way; see Figure 1 for sample stimuli). CIELAB is considered to be a perceptually uniform color space, meaning that a change in one unit is equivalent in perceptual magnitude across the entire space. A change of $\mathrm{a}^{*}=5$ reflects a suprathreshold color difference (the average $\Delta \mathrm{a}^{*}$ threshold for facial redness discrimination is $0.67, \mathrm{SEM}=0.16$, according to data from Re et al., 2011), meaning that participants should have been able to clearly perceive the color differences between the faces. Lightness, yellowness, hair, eyes, clothing, and background remained constant for each target.

\section{Results and discussion}


Selections of faces with increased redness were coded as 1, and selections of faces with decreased redness were coded as 0 for each trial. Selections for each participant were averaged across all six trials (separately for attractiveness and sexual attractiveness), resulting in proportions of increased redness selections over decreased redness selections (ranging from 0-1) for each dependent variable.

A one-sample t-test was performed against 0.5 (chance), with the proportion of redder face selections for attractiveness as the dependent variable. The results showed that redder faces were selected $(M=0.69, S D=0.32)$ significantly above chance level, $t(107)=6.12, p<.001, d$ $=.59$, indicating that participants perceived increased facial redness as more attractive than decreased facial redness.

We conducted another one-sample t-test with the proportion of redder face selections for sexual attractiveness. The results showed that redder faces were selected $(M=0.64, S D=0.33)$ significantly above chance level, $t(107)=4.37, p<.001, d=.42$, indicating that participants perceived increased facial redness as more sexually attractive than decreased facial redness.

These results provide initial support for our hypotheses. Women perceived men with increased (relative to decreased) facial redness as more attractive and sexually attractive. The question remains, however, whether an increase in facial redness is enhancing attractiveness, or whether a decrease in redness is diminishing attractiveness. We address this issue of directionality in Experiment 2.

\section{Experiment 2}

In Experiment 2, we investigate the specific direction of the effect observed in the previous experiment. It is possible that increased facial redness bolsters attractiveness or that decreased facial redness undermines attractiveness. Furthermore, it is possible that both of these 
relationships exist simultaneously. We utilize a neutral comparison condition in the present experiment to more thoroughly examine these possibilities.

\section{Method}

Participants. One hundred and nineteen women (mean age $=32.5$, range $=19-62)$ were recruited on Amazon's Mechanical Turk and paid \$.10 for participating. Our sample was not restricted by geographic area, but most of our participants (70\%) were from the United States.

Procedure. The procedure was very similar to that of Experiment 1, but with one notable change: Participants made four types of judgments rather than two. Specifically, all participants judged the attractiveness and sexual attractiveness of the six pairs of faces for two separate comparison types: increased redness versus neutral and decreased redness versus neutral. As a consequence, the difference in redness units between faces (i.e., original image vs. original image plus $\mathrm{a}^{*}=5$ ) was much smaller than the difference in redness units from the previous experiment (i.e., original image plus $\mathrm{a}^{*}=5$ vs. original image minus $\mathrm{a}^{*}=5$ ). The order of presentation across the six face pairs was randomized, and the order of the color-comparison types was randomized. Participants judged all faces for both comparison types on attractiveness, then repeated the procedure judging sexual attractiveness.

\section{Results and discussion}

Selections of faces with higher redness were coded as 1 , and selections of faces with lower redness were coded as 0 for each trial. Thus, neutral faces were sometimes coded as 1 or 0 , depending on whether they were being compared to a face with increased redness or decreased redness. Selections for each participant were averaged across trials within comparison type and rating type. This lead to four separate proportion scores for each participant: two for increased redness versus neutral (attractiveness and sexual attractiveness), and two for decreased redness 
versus neutral (attractiveness and sexual attractiveness). A series of one-sample t-tests were conducted (against a value of .5), one for each question type at each level of comparison.

Attractiveness. For the increased redness versus neutral comparison, participants were more likely than chance to select the redder faces as more attractive $(M=.63, S D=.31), t(118)=$ $4.45, p<.001, d=.42$. For the decreased redness versus neutral comparison, participants were also more likely than chance to select the redder (i.e., neutral) faces $(M=.83, S D=.26), t(118)=$ $14.22, p<.001, d=1.27$.

Sexual attractiveness. For the increased redness versus neutral comparison, participants were more likely than chance to select the redder faces as more sexually attractive $(M=.58, S D$ $=.36), t(118)=2.58, p=.011, d=.22$. For the decreased redness versus neutral comparison, participants were also more likely than chance to select the redder (i.e., neutral) faces $(M=.79$, $S D=.29), t(118)=10.83, p<.001, d=1.00$.

In additional analyses, we tested whether the effect for the increased redness versus neutral comparisons was different from the decreased redness versus neutral comparisons. Furthermore, we tested whether there was any difference between general attractiveness and sexual attractiveness ratings. We conducted a repeated measures ANOVA with two withinsubjects factors (type of color-comparison and type of rating) on the proportion of trials the redder face was selected. A main effect of comparison type emerged, $F(1,118)=66.88, p<$ .001 , indicating that the effect was stronger for the decreased redness versus neutral comparison $(M=.81)$, relative to the increased redness versus neutral comparison $(M=.61)$. A main effect of rating type also emerged, $F(1,118)=5.60, p=.020$, indicating that the effect of facial redness was stronger for general attractiveness $(M=.73)$, relative to sexual attractiveness $(M=.69)$. No interaction was observed between comparison type and response type $(F=.04, p=.84)$. 
The results of Experiment 2 provide information regarding the direction of the redattraction effect, with increased redness bolstering perceived attractiveness and decreased redness reducing perceived attractiveness, relative to a neutral baseline. This was the case for general attractiveness and sexual attractiveness, though the effects were stronger for general attractiveness.

Finally, the effect of decreased facial redness undermining attractiveness was stronger than the effect of increased facial redness enhancing attractiveness, indicating that the absence of facial redness may be considered particularly unattractive. This finding might demonstrate that the avoidance of a potentially unhealthy mate is more salient than the preference for an extremely healthy mate. This explanation would be consistent with the notion that avoidancebased motivation is often stronger than approach-based motivation in affective and cognitive systems (Baumeister, Bratslavsky, Finkenauer, \& Vohs, 2001; Cacioppo, Gardner, \& Berntson, 1999) as well as in social impressions (Schaller, 2008). Such a negativity bias carries adaptive value in the evolutionary context of social-impression formation; underestimating the negative qualities of social targets (and consequently engaging in approach behavior) carries more potential risk than underestimating their positive qualities (and consequently engaging in avoidance behavior). Therefore, particular vigilance to potentially negative social information is adaptively favorable (Nesse, 2005).

\section{Experiment 3}

In Experiment 3, we broadened our investigation to focus to the potential effect of facial redness on perceived healthiness. We also examined whether perceived healthiness would mediate the effect of facial redness on perceived attractiveness. In other words, the question of whether the effect of facial redness on perceived attractiveness could be explained by increases 
in perceived healthiness was our central focus. We utilized a controlled laboratory setting to ensure uniformity of color presentation, and we implemented a continuous measure of attractiveness in lieu of a forced choice design. Finally, we restrained our focus to increased facial redness versus a neutral baseline.

\section{Method}

Participants. One hundred and twenty six women were recruited from a university in the northeastern United States. Data on participant age were not collected in Experiment 3.

Procedure. The procedure was similar to that of the previous experiments, but with several important distinctions. First, we substituted the forced choice response option with 9point scales in the form of a slider (anchored from "not at all" to "very much") for each face. This allowed participants to make individual ratings for each face instead of having to choose one or the other. Second, we added another set of trials assessing perceived healthiness ("How healthy does this face look?"). Following perceived healthiness ratings, participants rated each face on perceived attractiveness ("How attractive does this face look?"). Composite scores were created by averaging the ratings for all 6 faces for each variable. The CIELAB values for facial stimuli were identical to those in the red/neutral comparison used in Experiment 2.

\section{Results and discussion}

We used MEMORE (Montoya \& Hayes, 2016) for SPSS to test the effect of facial redness on attractiveness. Ninety-five percent confidence intervals were generated from 5,000 bootstrap samples and are reported in brackets for each result below. Color type was entered as a dummy coded variable $(1=$ increased redness; $0=$ neutral baseline $)$. Perceived healthiness ratings were entered as the mediating variable, and perceived attractiveness was the dependent variable. 
Total effect (c path). The total effect of increased redness on perceived attractiveness was significant, $(c=.59[.38, .81], \mathrm{p}<.001)$, meaning that the faces with increased redness, on average, were rated as .59 points higher on attractiveness than neutral faces.

Individual effects ( $a$ path is the effect of the IV on the mediator; $b$ path is the effect of the mediators on the $D V)$. The faces with increased redness were perceived as healthier $(a=1.20$ $[.91,1.48], p<.001)$ than the neutral faces. Furthermore, perceived healthiness was positively related to perceived attractiveness (controlling for the effect of redness; $b=.50[.40, .60], p<$ .001 ; see Figure 2).

Indirect effect (a path*b path). The indirect effect of facial redness on perceived attractiveness via perceived healthiness was significant $(a b=.60[.40, .85], p<.001)$.

Direct effect (c path after accounting for the influence of mediating variable). The direct effect of facial redness on perceived attractiveness (controlling for the influence of perceived healthiness) was essentially zero $\left(c^{\prime}=-.01[-.21, .20], p=.94\right)$, indicating that perceived healthiness accounted for nearly all of the variance in the red-attraction relation.

The results of Experiment 3 provide further support for our hypotheses. Specifically, male faces with increased facial redness were perceived as healthier than the neutral faces, and perceived healthiness mediated the effect of increased facial redness on perceived attractiveness. In other words, the relationship between facial redness and perceived attractiveness could be attributed to increases in perceived healthiness.

\section{Experiment 4}

In Experiment 4, we aimed to replicate the results of the previous experiment while ruling out other potential mediating variables that may account for the red-attraction relation. We assessed perceptions of honesty, intelligence, and political conservatism in addition to 
healthiness and attractiveness. Honesty and intelligence are both valued characteristics for women rating men (Buss, 2015), but neither is strongly conceptually related to facial redness. If reddened faces are perceived as more honest and intelligent (and thus more attractive), this might indicate the presence of a halo effect, such that reddened faces are viewed more positively in general, rather than more healthy, specifically. It is feasible that redness may influence perceived political conservatism due to red being associated with the conservative political party in the United States. However, there is no reason to believe conservatism would be associated with attractiveness per se. Including these additional variables will help elucidate whether the effect of facial redness is specific to perceived healthiness, increases perceptions of positive characteristics in general, or increases perceptions of any characteristic.

\section{Method}

Participants. One hundred and sixty seven women (mean age $=19.6$, range $=18-23)$ were recruited from a university in the northeastern United States and compensated with extra course credit for participating. Ten participants provided missing data and were excluded from analyses.

Procedure. The procedure was similar to that of Experiment 3, but with the following changes. We added additional trials assessing perceived honesty ("How honest does this face look?"), perceived intelligence ("How intelligent does this face look?"), and political conservatism ("How politically conservative does this face look?"). Participants completed trials in blocks for each potential mediator, followed by a block of perceived attractiveness ratings. Composite scores were created across all six faces for each variable at each color level (see Tables 1 and 2 for descriptive statistics and correlations, respectively).

\section{Results}


We used MEMORE (Montoya \& Hayes, 2016) for SPSS to test the effect of facial redness on attractiveness through several parallel mediators. MEMORE tests for the indirect effect of each potential mediator while controlling for the indirect effects of the others. It also allows the size of each indirect effect to be compared directly, providing useful information regarding whether any one effect is stronger than another (Montoya \& Hayes, 2016). Ninety-five percent confidence intervals were generated from 5,000 bootstrap samples and are reported in brackets for each result below. Color condition was entered as a dummy coded variable $(1=$ increased redness; $0=$ neutral). Perceived healthiness, honesty, intelligence, and political conservatism were entered as mediators, and perceived attractiveness was the dependent variable.

Total effect. The total effect of increased redness on perceived attractiveness was significant, $(c=.36[.19, .53], p<.001)$, meaning that the faces with increased redness, on average, were rated as .36 points higher on attractiveness than the neutral faces.

Individual paths (a paths are the effects of the IV on the mediators; $b$ paths are the effects of the mediators on the $D V)$. The faces with increased redness were perceived as healthier $\left(a_{1}=\right.$ $1.34[1.08,1.60], p<.001)$ and more honest $\left(a_{4}=.29[.11, .47], p=.001\right)$ than the neutral faces (see Figure 3). No significant difference was observed for perceived intelligence $\left(a_{2}=.13[-.06\right.$, $.31], p=.18)$. Increased facial redness decreased perceived political conservatism $\left(a_{3}=-.21[-\right.$ $.41,-.01], p=.04)$.

Perceived healthiness was positively related to perceived attractiveness (controlling for the effect of redness and the other mediators; $\left.b_{1}=.28[.18, .38], p<.001\right)$. A similar relation was found between perceived intelligence and perceived attractiveness $\left(b_{2}=.14[.01, .28], p=.04\right)$, 
and perceived honesty and attractiveness $\left(b_{4}=.15[.01, .30], p=.03\right)$. No significant relation was observed between perceived conservatism and attractiveness $\left(b_{3}=-.01[-.13, .11], p=.94\right)$. Indirect effects (a paths $*$ b paths). The indirect effect of increased facial redness on perceived attractiveness via perceived healthiness was significant $\left(a_{1} b_{1}=.38[.21, .55], p<\right.$ .001). None of the other indirect effects were significantly different from zero (intelligence, $a_{2} b_{2}$ $=.02[-.02, .06], p=.32 ;$ conservatism, $a_{3} b_{3}=.00[-.05, .04], p=.99 ;$ honesty, $a_{4} b_{4}=.05[-.01$, $.12], p=.10$. Next, we compared the size of each indirect effect to determine whether the path through perceived healthiness differed from the other indirect paths. Pairwise contrasts of each indirect effect showed that perceived healthiness significantly differed from all three of the other potential mediators, and that perceived intelligence, honesty, and conservatism did not significantly differ from each other (see Table 3).

Direct effect (c path after accounting for the influence of mediating variables). The direct effect of increased facial redness on perceived attractiveness (controlling for the influence of the mediators) was not significantly different from zero $\left(c^{\prime}=-.08[-.27, .11], p=.40\right)$, indicating that perceived healthiness accounted for most of the variance in the red-attraction relation.

The results of Experiment 4 provide further support for our hypotheses. Facial redness was positively associated with male attractiveness, and this relationship was mediated by perceived healthiness. None of the other candidate mediator variables (honesty, intelligence, conservatism) had any mediating relationship between facial redness and attractiveness.

\section{General Discussion}

The results of the current studies support the notion that male facial redness positively influences heterosexual females' ratings of attractiveness, sexual attractiveness, and healthiness. Importantly, perceived healthiness was shown to mediate the effect of male facial redness on 
female perceptions of attractiveness, and this relationship was independent from other candidate mediators.

Faces convey a wide range of important cues that are detected and interpreted as social information to influence person perception (Perrett, 2010). The current research shows that facial coloration on male targets can be interpreted by women to indicate healthiness information, which influences perceptions of attractiveness. These findings show that the red-attractiveness effect that has been previously documented (Elliot et al., 2010; Roberts et al., 2010) extends to facial redness, and that healthiness as a mediator for men viewing women (Pazda et al., 2016) is relevant in the opposite direction (i.e., for women viewing men). Broadly, the findings contribute to the emerging literatures that explore the red-attractiveness effect (see Pazda \& Greitemeyer, 2015, for a review), and highlight the importance of facial coloration as a social cue (see Stephen $\&$ Perrett, 2015, for a review).

The current research, coupled with our prior research (Pazda et al., 2016), shows that changes in facial redness can influence both men's and women's perceived healthiness and attractiveness in the opposite sex, in broadly similar ways. However, facial redness is a sexually dimorphic characteristic, with men's faces typically being redder than women's (Frost, 2005). Given that sexually dimorphic information contributes to perceived attractiveness (Rhodes, 2006), we might expect larger effects for male targets than for female targets. On the other hand, male facial redness also contributes to perceptions of aggression, dominance, and anger (Young et al., 2013; Stephen et al., 2012), which might negatively influence attractiveness ratings. It is conceivable that men's facial redness conveys both desirable (e.g., health, masculinity) and undesirable (e.g., aggression) information, which contributes to attractiveness judgments in opposite ways, resulting in modest effects. Although it is not appropriate to directly compare 
effect sizes for the present findings and those of Pazda et al. (2016), subsequent work that equates male and female target stimuli on the focal characteristics (e.g., healthiness, attractiveness), amount of redness change across stimuli, and number of potential mediators, would be welcomed in this regard.

While we focused on the influence of facial color on enduring characteristics, facial color is also associated with changes in transient states. For instance, facial redness is associated with anger and sexual excitation, and it is likely that facial redness also influences social perceptions related to such states. Future work should investigate whether face color influences judgments uniformly, the role of context in making color-based social judgments, and the boundaries of facial color change necessary to influence social judgments.

In our work, we presented facial color that changed in increments of CIELAB $\mathrm{a}^{*}=5$. This difference might be considered a subtle value relative to the gamut of human color perception, but it represents a difference that greatly exceeds the threshold for visual detection displayed on face stimuli (Re et al., 2011). This is important because there should be no question of whether the participant detected the differences. However, the relationship between actual physical health and quantitative amount of redness change in human faces is not known. Future work, for instance, could determine how much physical exercise is needed to correspond to an 'attractive' change in facial redness.

The current research has limitations that can be considered for future work. First, Experiments 1 and 2 were conducted online, which precluded color presentation from being rigorously controlled. Experiments 3 and 4 were conducted in a laboratory environment with color-calibrated monitors in order to address this limitation. While these four studies produced consistent results, web-based color research should be considered with this limitation in mind. 
Second, all of the stimulus pictures used herein were of young Caucasian men, which prohibits the generalizability of our findings to older men or men of different ethnic backgrounds. We would expect to see similar results across targets with various demographic backgrounds, but the results may be weaker and future research is necessary before drawing any definitive conclusions. Similarly, each of our targets displayed a neutral facial expression. It is possible that facial redness is perceived as healthy in the absence of an expressed emotion, but fails to do so when perceived on an angry face, for example. Adding facial expression as a variable may shine light on potential boundary conditions of the red effect. Finally, our manipulations of facial redness exceeded human visual detection thresholds, but they were still relatively small $\left(\Delta \mathrm{a}^{*}=\right.$ 5). It remains unclear whether increasing facial redness at higher levels may lead to an opposite pattern of results. For example, extreme redness may undermine attractiveness perceptions because it is associated with anger or fever. Future research utilizing a wider gamut of facial redness could elucidate this possibility.

Facial redness is often manipulated globally (i.e., across the entire face) to study the influence of facial color on social perception, as in the current work. However, it is likely the case that socially relevant cues (e.g., health, attractiveness) manifest selectively (i.e., patterns of color changes in specific facial regions). For instance, Jones et al. (2016) found that redness on the cheeks, but not on other areas of the face, were important when judging perceived health. Additionally, global redness is not the only dimension through which facial color can influence health perceptions. Global yellowness (Jones et al., 2016; Stephen, Law Smith, Stirrat, \& Perrett, 2009), lightness (Stephen et al., 2009), chromatic and luminance contrast (Russell, Porcheron, Sweda, Jones, Mauger, \& Morizot, 2016), and periorbital luminance (Jones et al., 2016), positively predict perceived health. Future research would do well to move toward studying the 
influence of localized facial color, and all dimensions of color (redness, yellowness, lightness), on perception and evaluation.

Previous research investigating the effect of red coloration on men's attractiveness has yielded mixed results. These mixed results may be a result of moderating variables, such as perceived aggressiveness (Stephen et al., 2012), anger (Wiedemann et al., 2015), incompetence (Maier et al., 2013), or numerous other possibilities. The present research found a red-attraction effect, but it is important to consider that our color manipulation was on the face, rather than on peripheral objects (e.g., clothing). Color may convey different information when displayed on human skin, relative to extraneous items, because reddened skin implies physiological changes (e.g., vascularization, oxygenation) that are not implied on external objects such as clothing. Thus, perceiving redness in the context of skin may lead to different perceptions (e.g., healthiness) than perceiving redness in other contexts.

Altogether, the current research highlights the important role of facial color as a social cue, capable of influencing evaluative judgments. Future research in this area can lead to better understanding how facial coloration is altered by psychological and physiological states, impacts a range of social judgements, and influences social communication. 


\section{References}

Armstrong, N., \& Welsman, J. (2001). Peak oxygen uptake in relation to growth and maturation in 11- to 17-year-old humans. European Journal of Applied Physiology, 85(6), 546-551. http://doi.org/10.1007/s004210100485

Baumeister, R. F., Bratslavsky, E., Finkenauer, C., \& Vohs, K. D. (2001). Bad is stronger than good. Review of general psychology, 5(4), 323.

Buechner, V. L., Maier, M. A., Lichtenfeld, S., \& Elliot, A. J. (2015). Emotion expression and color: their joint influence on perceived attractiveness and social position. Current Psychology, 34(2), 422-433. http://doi.org/10.1007/s12144-014-9266-x

Buss, D. (2015). Evolutionary psychology: The new science of the mind. Psychology Press.

Cacioppo, J. T., Gardner, W. L., \& Berntson, G. G. (1999). The affect system has parallel and integrative processing components: Form follows function. Journal of Personality and Social Psychology, 76(5), 839-855. doi.org:10.1037/0022-3514.76.5.839

Carrito, M., dos Santos, I. M. B., Lefevre, C. E., Whitehead, R. D., da Silva, C. F., \& Perrett, D. I. (2016). The role of sexually dimorphic skin colour and shape in attractiveness of male faces. Evolution and Human Behavior, 37(2), 125-133.

Changizi, M., \& Shimojo, S. (2011). Social Color Vision. In R. B. Adams (Ed.), The Science of Social Vision. Oxford University Press.

Changizi, M., Zhang, Q., \& Shimojo, S. (2006). Bare skin, blood and the evolution of primate colour vision. Biology Letters, 2(2), 217-21. http://doi.org/10.1098/rsbl.2006.0440

Charkoudian, N. (2003). Skin blood flow in adult human thermoregulation: how it works, when it does not, and why. Mayo Clinic Proceedings, 78(5), 603-12. http://doi.org/10.4065/78.5.603 
Edwards, E. A., \& Duntley, S. Q. (1939). The pigments and color of living human skin. American Journal of Anatomy, 65(1), 1-33. http://doi.org/10.1002/aja.1000650102

Elliot, A. J., \& Maier, M. A. (2013). The Red-Attractiveness Effect, Applying the Ioannidis and Trikalinos (2007b) Test, and the Broader Scientific Context: A Reply to Francis (2013), 142(1), 297-300. http://doi.org/10.1037/a0029592

Elliot, A. J., \& Niesta, D. (2008). Romantic red: red enhances men's attraction to women. Journal of Personality and Social Psychology, 95(5), 1150-64. http://doi.org/10.1037/00223514.95 .5 .1150

Elliot, A. J., \& Maier, M. A. (2012). Color-in-context theory. Advances in experimental social psychology, 45, 61-125. doi: 10.1016/B978-0-12-394286-9.00002-0

Elliot, A. J., Niesta, D., Greitemeyer, T., Lichtenfeld, S., Gramzow, R. H., Maier, M. A., \& Liu, H. (2010). Red, rank, and romance in women viewing men. Journal of Experimental Psychology: General, 139(3), 399-417. http://doi.org/10.1037/a0019689

Frost, P. (2005). Fair women, dark men: The forgotten roots of racial prejudice. Lisa Loucks Christenson Publishing, Minnesota, United States.

Gilston, A., \& Privitera, G. J. (2016). A "healthy" color: Information about healthy eating attenuates the "red effect". Global Journal of Health Science, 8, 56-61. doi10.5539/gjhs.v8n1p56.

Guéguen, N. (2012a). Color and women attractiveness: When red clothed women are perceived to have more intense sexual intent. The Journal of Social Psychology, 152(3), 261-265. doi:abs/10.1080/00224545.2011.605398

Guéguen, N. (2012b). Color and women hitchhikers' attractiveness: Gentlemen drivers prefer red. Color Research and Application, 37(1), 76-78. 
Guéguen, N., \& Jacob, C. (2012a). Clothing color and tipping: Gentlemen patrons give more tips to waitresses with red clothes. Journal of Hospitality \& Tourism Research, doi:1096348012442546.

Guéguen, N., \& Jacob, C. (2012b). Lipstick and tipping behavior: When red lipstick enhance waitresses tips. International Journal of Hospitality Management, 31(4), 1333-1335.

Hesslinger, V. M., Goldbach, L., \& Carbon, C. C. (2015). Men in red: A reexamination of the red-attractiveness effect. Psychonomic bulletin \& review, 22(4), 1142-1148.

Hutchings, J. (2004). Colour in folklore and tradition-The principles. Color Research \& Application, 29(1), 57-66. http://doi.org/10.1002/col.10212

Johnson, J. M. (1998). Physical training and the control of skin blood flow. Medicine and Science in Sports and Exercise, 30(3), 382-386.

Jones, A. L., Porcheron, A., Sweda, J. R., Morizot, F., \& Russell, R. (2016). Coloration in different areas of facial skin is a cue to health: The role of cheek redness and periorbital luminance in health perception. Body Image, 17, 57-66. doi:10.1016/j.bodyim.2016.02.001

Knight, C., Power, C., \& Watts, I. (1995). The human symbolic revolution: a Darwinian account. Cambridge Archaeological Journal, 5(1), 75-114.

Lin, H. (2014). Red-colored products enhance the attractiveness of women. Displays, 35, 202205. doi: 10.1016/j.displa.2014.05.009

Lynn, M., Giebelhausen, M., Garcia, S., Li, Y., \& Patumanon, I. (2013). Clothing Color and Tipping: An Attempted Replication and Extension. Journal of Hospitality \& Tourism Research, 1-9. Doi:10.1177/1096348013504001

Panza, J. A., Quyyumi, A. A., Brush, J. E., \& Epstein, S. E. (1990). Abnormal endotheliumdependent vascular relaxation in patients with essential hypertension. The New England 
Journal of Medicine, 323(1), 22-7. http://doi.org/10.1056/NEJM199007053230105

Maier, M. A., Elliot, A. J., Lee, B., Lichtenfeld, S., Barchfeld, P., \& Pekrun, R. (2013). The influence of red on impression formation in a job application context. Motivation and Emotion, 37(3), 389-401.

Montoya, A. K., \& Hayes, A. F. (2016, June 30). Two condition within-participant statistical mediation analysis: A path-analytic framework. Psychological Methods. Advance online publication. doi: $\underline{10.1037 / \text { met0000086 }}$

Nesse, R. M. (2005). Natural selection and the regulation of defenses: A signal detection analysis of the smoke detector principle. Evolution and Human Behavior, 26, 88-105.

Nestor, A., \& Tarr, M. J. (2008). Gender recognition of human faces using color. Psychological Science, 19(12), 1242-1246.

Pazda, A. D., Elliot, A. J., \& Greitemeyer, T. (2014). Perceived sexual receptivity and fashionableness: Separate paths linking red and black to perceived attractiveness. Color Research \& Application, 39(2), 208-212. http://doi.org/10.1002/col.21804

Pazda, A. D., \& Greitemeyer, T. (2015). Color in romantic contexts in humans. In A. Elliot, M. Fairchild, \& A. Franklin (Eds.), Handbook of color psychology. Cambridge, England: Cambrdige University Press.

Pazda, A. D., Thorstenson, C. A., Elliot, A. J., \& Perrett, D. I. (2016). Womens Facial Redness Increases Their Perceived Attractiveness: Mediation Through Perceived Healthiness. Perception. http://doi.org/10.1177/0301006616633386

Perrett, D. (2010). In your face: The new science of human attraction. Palgrave Macmillan. Ponsonby, A.-L., Dwyer, T., \& Couper, D. (1997). Sleeping Position, Infant Apnea, and Cyanosis: A Population-based Study. Pediatrics, 99(1), e3-e3. 
http://doi.org/10.1542/peds.99.1.e3

Prokop, P., Pazda, A. D., \& Elliot, A. J. (2015). Influence of conception risk and sociosexuality on female attraction to male red. Personality and Individual Differences, 87, 166-170. http://doi.org/10.1016/j.paid.2015.07.042

Ragas, M. C., \& Kozlowski, K. (1998). Read My Lips: A Cultural History of Lipstick. Chronicle, 126.

Re, D. E., Whitehead, R. D., Xiao, D., \& Perrett, D. I. (2011). Oxygenated-blood colour change thresholds for perceived facial redness, health, and attractiveness. PloS One, 6(3), e17859. http://doi.org/10.1371/journal.pone.0017859

Rhodes, G. (2006). The evolutionary psychology of facial beauty. Annual Review of Psychology, 57, 199-226. http://doi.org/10.1146/annurev.psych.57.102904.190208

Roberts, S. C., Owen, R. C., \& Havlicek, J. (2010). Distinguishing between perceiver and wearer effects in clothing color-associated attributions.Evolutionary Psychology, 8(3), doi:147470491000800304.

Russell, R., Porcheron, A., Sweda, J., Jones, A., Mauger, E. \& Morizot, F. (2016). Facial contrast is a cue for perceiving health from the face. Journal of Experimental Psychology: Human Perception and Performance, 42(9), 1354-1362.

Said, C. P., \& Todorov, A. (2011). A Statistical Model of Facial Attractiveness. Psychological Science, 22(9), 1183-1190. http://doi.org/10.1177/0956797611419169

Schaller, M. (2008). Evolutionary bases of first impressions. In N. Ambady \& J. J. Skowronski (Eds.), First impressions (pp. 15-34). New York, NY: Guilford.

Stephen, I. D., Coetzee, V., Law Smith, M., \& Perrett, D. I. (2009). Skin blood perfusion and oxygenation colour affect perceived human health. PloS One, 4(4), e5083. 
http://doi.org/10.1371/journal.pone.0005083

Stephen, I. D., Law Smith, M. J., Stirrat, M. R., \& Perrett, D. I. (2009). Facial skin coloration affects perceived health of human faces. International Journal of Primatology, 30(6), 845857.

Stephen, I. D., \& McKeegan, A. M. (2010). Lip colour affects perceived sex typicality and attractiveness of human faces. Perception, 39(8), 1104-1110. http://doi.org/10.1068/p6730

Stephen, I. D., Oldham, F. H., Perrett, D. I., \& Barton, R. (2012). Redness Enhances Perceived Aggression, Dominance and Attractiveness in Men's Faces. Evolutionary Psychology, 10(3), 562-572.

Stephen, I. D., \& Perrett, D. I. (2015). Color and face perception. In A. Elliot, M. Fairchild, \& A. Franklin (Eds.), Handbook of color psychology. Cambridge, England: Cambrdige University Press.

Tan, K. W., \& Stephen, I. D. (2013). Colour detection thresholds in faces and colour patches. Perception, 42(7), 733-741. http://doi.org/10.1068/p7499

Tarr, M. J., Kersten, D., Cheng, Y., \& Rossion, B. (2010). It's Pat! Sexing faces using only red and green. Journal of Vision, 1(3), 337-337. http://doi.org/10.1167/1.3.337

Wiedemann, D., Burt, D. M., Hill, R. A., \& Barton, R. A. (2015). Red clothing increases perceived dominance, aggression and anger. Biology letters, 11(5), 20150166.

Young, S. G., Elliot, A. J., Feltman, R., \& Ambady, N. (2013). Red enhances the processing of facial expressions of anger. Emotion, 13(3), 380-4. http://doi.org/10.1037/a0032471

Young, S. G. (2015). The effect of red on male perceptions of female attractiveness: Moderation by baseline attractiveness of female faces. European Journal of Social Psychology, 45(2), 146-151. 

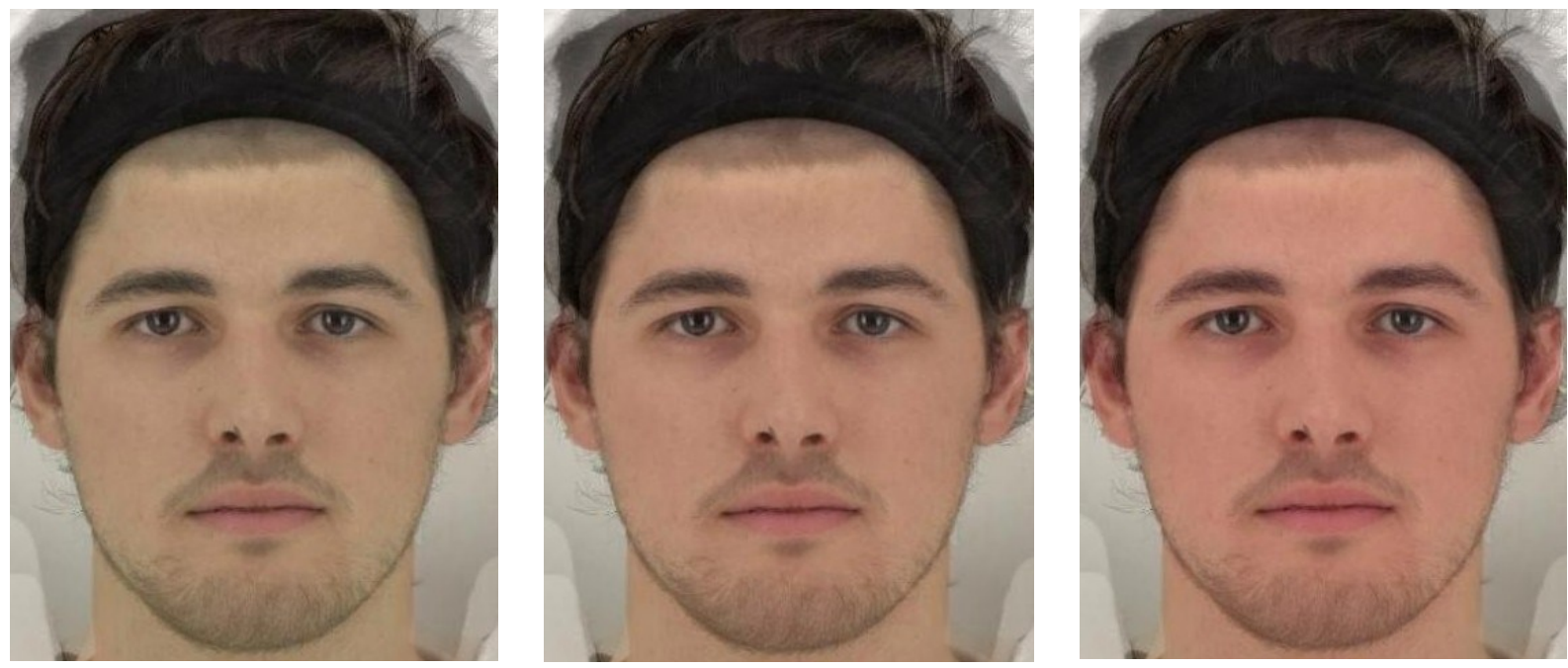

Figure 1. Example of the face stimuli used. Faces were manipulated on the CIELAB a* (redness) color axis by -5 units (left) or +5 units (right). 


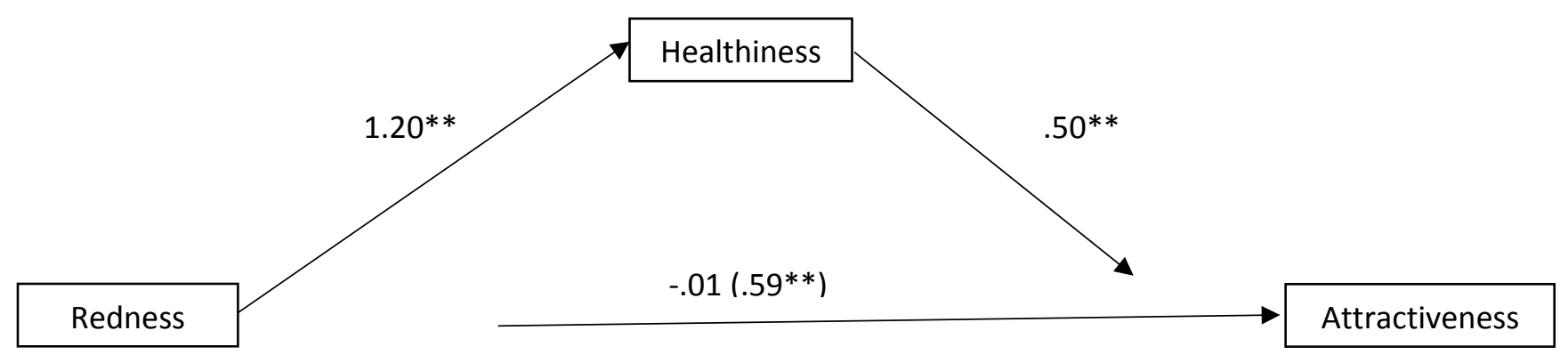

Figure 2. $* * p<.001$. Effect of increased redness on perceived attractiveness through perceived healthiness in Experiment 3. Coefficients are unstandardized estimates, and the coefficient in parentheses is the total effect. The indirect effect is .60, 95\% $\mathrm{CI}=[.40, .85]$. 


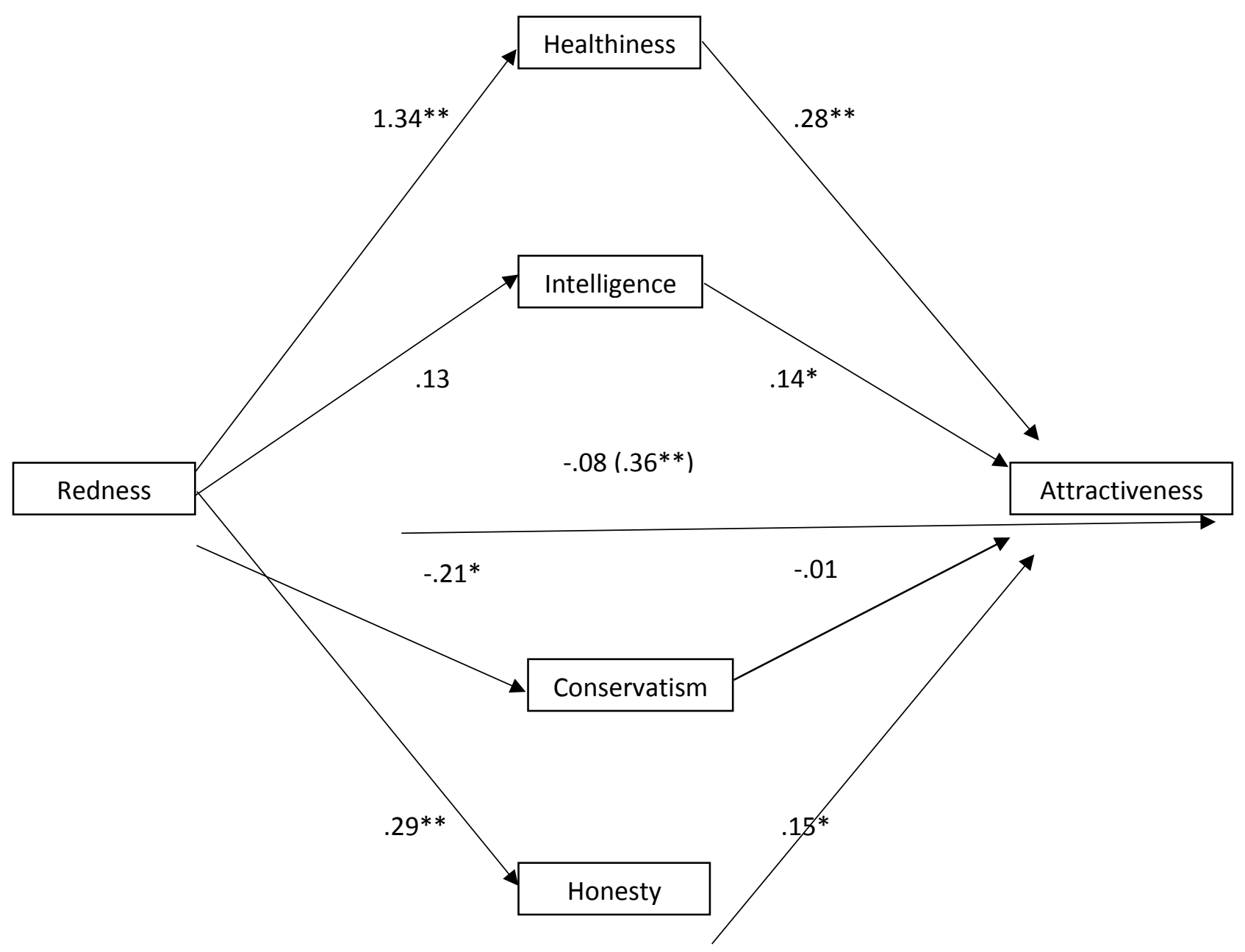

Figure 3. ${ }^{*} p<.05 ; * * p<.001$. Effects of increased redness on perceived attractiveness through four potential mediators in Experiment 4. Coefficients are unstandardized estimates, and the coefficient in parentheses is the total effect. Indirect effects of red on perceived attractiveness through each potential mediator [95\% confidence intervals in brackets] are as follows.

Healthiness: $.38[.21, .55]$; Intelligence: .02 [-.02, .06]; Conservatism: .00 [-.05, .04]; Honesty: $.05[-.01, .12]$. 
Table 1. Descriptive statistics for variables in Experiment 4 averaged across faces and color conditions.

$\begin{array}{llll} & \text { Mean } & \text { Standard deviation } & \text { Range } \\ \text { Healthiness } & 5.48 & 1.03 & 2.8-8.8 \\ \text { Intelligence } & 4.99 & 0.95 & 2.3-7.4 \\ \text { Conservatism } & 4.70 & 1.12 & 1.8-7.8 \\ \text { Honesty } & 4.87 & 1.13 & 1.7-9.0 \\ \text { Attractiveness } & 4.65 & 1.12 & 1.9-8.0\end{array}$

Table 2. Correlations between variables in Experiment 4.

$$
\text { Intelligence Conservatism Honesty Attractiveness }
$$

$\begin{array}{lllll}\text { Healthiness } & .48 & .07 & .58 & .39\end{array}$

$\begin{array}{lllll}\text { Intelligence } & -- & .28 & .52 & .31\end{array}$

$\begin{array}{llll}\text { Conservatism -- } & -- & .23 & .22\end{array}$

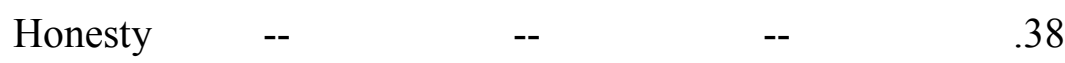

Table 3. Pairwise contrasts of specific indirect effects in Experiment 4.

$\begin{array}{lcccc} & \text { Effect } & \text { SE } & \text { Lower CI } & \text { Upper CI } \\ \text { Healthiness vs intelligence } & .36 & .09 & .19 & .53 \\ \text { Healthiness vs conservatism } & .37 & .09 & .19 & .55 \\ \text { Healthiness vs honesty } & .33 & .09 & .16 & .51 \\ \text { Intelligence vs conservatism } & .02 & .03 & -.04 & .07 \\ \text { Intelligence vs honesty } & -.03 & .04 & -.12 & .05 \\ \text { Conservatism vs honesty } & -.04 & .04 & -.13 & .03\end{array}$

